\title{
The structure of liquid GeSe revisited: A first principles molecular dynamics study
}

Sébastien Le Roux, Assil Bouzid, Mauro Boero, and Carlo Massobrio

Citation: The Journal of Chemical Physics 138, 174505 (2013); doi: 10.1063/1.4803115

View online: https://doi.org/10.1063/1.4803115

View Table of Contents: http://aip.scitation.org/toc/jcp/138/17

Published by the American Institute of Physics

\section{Articles you may be interested in}

GeSe monolayer semiconductor with tunable direct band gap and small carrier effective mass Applied Physics Letters 107, 122107 (2015); 10.1063/1.4931459

Giant piezoelectricity of monolayer group IV monochalcogenides: SnSe, SnS, GeSe, and GeS Applied Physics Letters 107, 173104 (2015); 10.1063/1.4934750

Nanosecond switching in GeSe phase change memory films by atomic force microscopy Applied Physics Letters 104, 053109 (2014); 10.1063/1.4863495

Effect of density of localized states on the ovonic threshold switching characteristics of the amorphous GeSe films

Applied Physics Letters 103, 042908 (2013); 10.1063/1.4816349

Threshold resistive and capacitive switching behavior in binary amorphous GeSe Journal of Applied Physics 111, 102807 (2012); 10.1063/1.4714705

Structure of amorphous GeSeg by neutron diffraction and first-principles molecular dynamics: Impact of trajectory sampling and size effects

The Journal of Chemical Physics 145, 084502 (2016); 10.1063/1.4961265

\section{AIP | the Jounnalof Chemical Physics}

PERSPECTIVES 


\title{
The structure of liquid GeSe revisited: A first principles molecular dynamics study
}

\author{
Sébastien Le Roux, Assil Bouzid, Mauro Boero, and Carlo Massobrio \\ Institut de Physique et de Chimie des Matériaux de Strasbourg, CNRS and University of Strasbourg, \\ UMR 7504, 23 rue du Loess, BP43, F-67034 Strasbourg Cedex 2, France
}

(Received 5 March 2013; accepted 15 April 2013; published online 3 May 2013)

\begin{abstract}
Early first-principles molecular dynamics results on liquid GeSe were characterized by shortcomings in the description of $\mathrm{Ge}-\mathrm{Ge}$ (and to a lesser extent $\mathrm{Se}-\mathrm{Se}$ ) short range correlations. In that case the exchange-correlation functional adopted was the one devised by Perdew and Wang (PW91). In the search of improvements in the atomic-scale modelling of this liquid, we have produced new sets of data by employing two different schemes for the exchange-correlation part within the density functional theory approach. The two functionals selected are those proposed by Becke, Lee, Yang, and Parr (BLYP) and by Perdew, Burke, and Ernzerhof (PBE). The PBE results turned out to be quite similar to the PW91 ones. The BLYP results feature instead a better account of the Ge-Ge first shell of neighbors, correctly exhibiting two clear maxima separated by a deep minimum. Due to the increase in the number of the tetrahedral structural units, the atomic mobility of $\mathrm{Ge}$ and Se atoms in the network is reduced with respect to the PW91 case. This brings the diffusion coefficients of the two species down to values close to those of liquid $\mathrm{Ge}_{2} \mathrm{Se}_{3}$ and liquid $\mathrm{GeSe}_{2}$. (C) 2013 AIP Publishing LLC. [http://dx.doi.org/10.1063/1.4803115]
\end{abstract}

\section{INTRODUCTION}

The study of structural features and bonding properties in chalcogenides liquids and glasses is motivated by experimental data underlying the appearance of intermediate range order (IRO) with changing composition, as found in $\mathrm{Ge}_{x} \mathrm{Se}_{1-x}$ $(0 \leq x \leq 1)$ systems within the $0.15 \lesssim x \lesssim 0.40$ range. ${ }^{1,2} \mathrm{Re}-$ liable atomic-scale models describing these networks are very useful to highlight the interplay between the coordination environment and the diffraction experiments. ${ }^{3}$ In the past, the absence of intermediate range order in liquid GeSe ( $l$-GeSe) was associated to the lack of a prevailing structural feature such as the tetrahedron. ${ }^{4,5}$ We recall that the results of Ref. 4 were obtained by using the Perdew-Wang (PW91) generalized gradient approximation (GGA) for the exchange correlation functional (XC) within density functional theory (DFT). ${ }^{6,7}$ Several coordinations environments were found to contribute, in close proportions, to the $\mathrm{Ge}$ and Se shells of neighbors, pointing out the disruption of the tetrahedral $\mathrm{GeSe}_{4}$-based network. Despite the impact of these findings, substantial lack of agreement with experiments for the $\mathrm{Ge}-\mathrm{Ge}$ correlations did exist, preventing uncontroversial assessment. In particular, the pattern consisting of two maxima clearly separated by a deep minimum could not be reproduced in the short range part of the pair distribution function $\mathrm{g}_{\mathrm{GeGe}}(\mathrm{r})$. In analogy with $\mathrm{GeSe}_{2}$, this outcome was attributed to an overestimate of the metallic character of bonding for intermediate $\mathrm{Ge}_{x} \mathrm{Se}_{1-x}$ compositions. ${ }^{4}$ The application of an alternative GGA scheme for the XC, such as the one due to the Becke (B) for the exchange energy and Lee, Yang, and Parr (LYP) for the correlation energy, led to a substantial improvement for the structural properties in the case of liquid and glassy $\mathrm{GeSe}_{2} \cdot{ }^{8-10}$ Specifically, $\mathrm{g}_{\mathrm{GeGe}}(\mathrm{r})$ took a more structured profile, exhibiting signatures (as the one associated to the homopolar bonds) absent in the previous PW91 results. Ever since the application of Becke, Lee, Yang, and Parr (BLYP) to liquid $\mathrm{GeSe}_{2}$, this same recipe proved successful also for other disordered $\mathrm{Ge}_{x} \mathrm{Se}_{1-x}$ systems on the Se-rich side of compositions ( $\mathrm{x} \lesssim 0.33$ ), as well as for liquid and glassy $\mathrm{Ge}_{2} \mathrm{Se}_{3}$ $(\mathrm{x}=0.4) .{ }^{11-13}$

In view of these considerations, it appears worthwhile to ascertain whether, in the case of liquid $\mathrm{GeSe}$, the level of agreement between theory and experiments could be improved by resorting to the BLYP XC scheme. For this purpose, and using this GGA approach, new first-principles molecular dynamics data (FPMD) have been produced over an extended time interval, at the same thermodynamic conditions adopted in Refs. 4 and 5. Due to the proved sensitivity to the XC functional of chalcogenides DFT models, we have also considered a third XC scheme, the Perdew-BurkeErnzerhof (PBE) one, largely employed in current simulations of binary and ternary disordered materials. ${ }^{15-18}$ Therefore, in this paper, a set of results based on the PBE functional is also made available.

\section{THEORETICAL MODEL}

Our simulations were performed on a $(N, V, T)$ ensemble on a system containing $N=120(60 \mathrm{Ge}$ and $60 \mathrm{Se})$ atoms (BLYP and PBE calculations) and $N=108$ (54 Ge and $54 \mathrm{Se}$ ) (PW91 calculations). We used periodically repeated cubic cells of $14.58 \AA$ (BLYP and PBE cases) and $14.08 \AA$ (PW91 case), corresponding, for the two box sizes, to the same experimental density of the liquid at a temperature $T=1000 \mathrm{~K}^{2}{ }^{2}$ The electronic structure was described within DFT and evolved self-consistently during the motion. ${ }^{19}$ A new trajectory was first produced for the PW91 case, with 
statistics accumulated over 100 ps. This new trajectory covered a longer time than the one considered in Ref. 4, thus providing a better statistics. Nonetheless, we observe that the structural properties resulting from the old and the new sets of data exhibit essentially no differences. Indeed, structural properties are expected to converge quicker than dynamical properties, which are by their own nature more sensitive to the simulation time. In fact, this is confirmed by the estimation of the diffusion coefficients, which, evaluated on a longer time interval, are found to be significantly smaller, due to the extended relaxation times inherent in the diffusion process. These effects were out of the reach of the previous simulations, carried out over $17 \mathrm{ps}$ and featuring a large statistical noise for $\mathrm{t}>5 \mathrm{ps}$. Further details are given in Sec. VI. Concerning the two additional GGA schemes employed, a few remarks are in order. In the BLYP case, we expect to observe a larger localization of the valence electrons than in the PW91 approach. ${ }^{14}$ This leads to structural and dynamical properties better reflecting the predominant tetrahedral character of these systems. As to the PBE scheme, we recall here that this GGA is closer in nature to the PW91 since it ensures a continuous extrapolation of the exchange-correlation energy to the homogeneous electron gas. However, it was designed to correct some of deficiencies affecting the original PW91 formula, such as the parametrization of the electronic gradients. ${ }^{15}$ For all of these reasons, it is desirable to get a better insight into the performance of BLYP and PBE for a bonding case characterized by a lower degree of ionicity (and a correspondingly higher metallic character) than the one of liquid and glassy $\mathrm{GeSe}_{2}$.

For $\mathrm{Ge}_{x} \mathrm{Se}_{1-x}$ liquids and glasses, the delicate balance between the ionic and the covalent character of bonding results in a high sensitivity to the specific exchange-correlation functional employed. This is due to the moderate difference of electronegativity between $\mathrm{Ge}$ and $\mathrm{Se}\left(\Delta_{e l}=0.54\right)$. For the sake of comparison, $\Delta_{e l}=1.52$ for $\mathrm{Si}$ and $\mathrm{O}$, that form the perfect tetrahedral network $\mathrm{SiO}_{2} \cdot{ }^{20}$ In view of the role played by $\Delta_{e l}$, the above rationale is not applicable to other tetrahedral systems, ruling out any generic correlation between the kind of short range motif and the sensitivity of a given system to the exchange-correlation functional adopted.

In our work, the valence electrons were treated explicitly, in conjunction with norm conserving pseudopotentials of the Trouiller-Martins type to account for core-valence interactions. ${ }^{21}$ The wave functions were expanded at the $\Gamma$ point of the supercell on a plane wave basis set with an energy cutoff $E_{c}=30 \mathrm{Ry}$. Within our first-principles molecular dynamics approach, we used a fictitious electron mass of 2000 a.u. (i.e., in units of $m_{e} a_{0}^{2}$ where $m_{e}$ is the electron mass and $a_{0}$ is the Bohr radius) and time steps of $\Delta t=0.12 \mathrm{fs}$ (BLYP, $\mathrm{PBE}$ ) and $\Delta t=0.24 \mathrm{fs}$ (PW91) to integrate the equations of motion. The initial coordinates for the BLYP model were provided by a configuration extracted from the fully equilibrated trajectories obtained for liquid $\mathrm{GeSe}_{2}$ in Ref. 23. To achieve the correct composition, the number of Ge atoms was changed to 60 by modifying the identity of 20 randomly chosen $\mathrm{Se}$ atoms.

To lose memory of the initial configuration, simulations were performed at $\mathrm{T}=2000 \mathrm{~K}$ and at $\mathrm{T}=1000 \mathrm{~K}$ over a time period of 50 ps. Statistical averages were taken over an equilibrium trajectory of $100 \mathrm{ps}$. Similarly, the PBE results were obtained by selecting an equilibrated BLYP configuration and by accumulating statistics over $100 \mathrm{ps}$, this interval following an initial $50 \mathrm{ps}$ (at $\mathrm{T}=2000 \mathrm{~K}$ and $\mathrm{T}=1000 \mathrm{~K}$ ) period necessary to lose memory of the starting BLYP configuration. ${ }^{22}$

For all of the three calculations carried out in the present work, statistical errors are obtained by partitioning the trajectory in 20 subtrajectories and calculating the standard deviation. Error bars are of the order of 5\% at most for structural properties, while they are larger for the diffusion coefficients, due to the long relaxation times needed to attain the stationary state for computing either the mean square displacement or the velocity-velocity autocorrelation function, from which diffusion constants are extracted.

\section{NEUTRON TOTAL STRUCTURE FACTOR AND TOTAL PAIR DISTRIBUTION FUNCTION}

The total neutron structure factor $S_{\mathrm{T}}(k)$ is defined by

$$
S_{\mathrm{T}}(k)-1 \equiv \sum_{\alpha=1}^{n} \sum_{\beta=1}^{n} \frac{c_{\alpha} c_{\beta} b_{\alpha} b_{\beta}}{\langle b\rangle^{2}}\left[S_{\alpha \beta}(k)-1\right],
$$

where $\alpha$ and $\beta$ denote the chemical species, $n=2$ is the number of different chemical species, $c_{\alpha}$ and $b_{\alpha}$ are the atomic fraction and coherent neutron scattering length of chemical species $\alpha,\langle b\rangle=c_{\mathrm{Ge}} b_{\mathrm{Ge}}+c_{\mathrm{Se}} b_{\mathrm{Se}}$ is the mean coherent neutron scattering length, and $S_{\alpha \beta}(k)$ is a Faber-Ziman partial structure factor. The coherent neutron scattering lengths for $\mathrm{Ge}$ and $\mathrm{Se}$ of natural isotopic abundance are $b_{\mathrm{Ge}}=8.185 \mathrm{fm}$ and $b_{\mathrm{Se}}$ $=7.970 \mathrm{fm} \cdot{ }^{2}$ The corresponding real space information is given by the total pair distribution function

$$
\begin{aligned}
g_{\mathrm{T}}(r)-1 & =\frac{1}{2 \pi^{2} n_{0} r} \int_{0}^{\infty} \mathrm{d} k k\left[S_{\mathrm{T}}(k)-1\right] \sin (k r) \\
& =\sum_{\alpha=1}^{n} \sum_{\beta=1}^{n} \frac{c_{\alpha} c_{\beta} b_{\alpha} b_{\beta}}{\langle b\rangle^{2}}\left[g_{\alpha \beta}(r)-1\right],
\end{aligned}
$$

where $n_{0}$ is the atomic number density and $g_{\alpha \beta}(r)$ is a partial pair distribution function.

In Fig. 1 the experimental total neutron structure factor $S_{\mathrm{T}}^{\exp }(k)^{2}$ is presented along with the calculated $S_{\mathrm{T}}^{\mathrm{th}}(k)_{\mathrm{PW} 91}$, $S_{\mathrm{T}}^{\text {th }}(k)_{\mathrm{BLYP}}$, and $S_{\mathrm{T}}^{\text {th }}(k)_{\mathrm{PBE}}$. We underscore here that $S_{\mathrm{T}}^{\text {th }}(k)$ is calculated directly in $k$-space and not obtained upon Fourier transform of real-space computed pair distribution functions. The first procedure has to be preferred over the second since no approximations related to the finite range of integration are introduced. However, the Fourier transform procedure has also been adopted in the literature to reduce the statistical noise, especially in the case of glassy configurations at room temperature. ${ }^{11}$ As a first indication, $S_{\mathrm{T}}^{\text {th }}(k)_{\mathrm{PW} 91}$ and $S_{\mathrm{T}}^{\mathrm{th}}(k)_{\mathrm{PBE}}$ appear to perform quite similarly, the reduced statistical noise inherent in $S_{\mathrm{T}}^{\text {th }}(k)_{\mathrm{PBE}}$ being due to the larger system and the much longer temporal trajectory. Adopting the BLYP approach has the effect of improving the intensity of the peak at $k \sim 4 \AA^{-1}$, while the height of the peak at $k \sim 2.2 \AA^{-1}$ is underestimated and its position is slightly shifted at lower $k$ values. The oscillating patterns of the three calculated total 


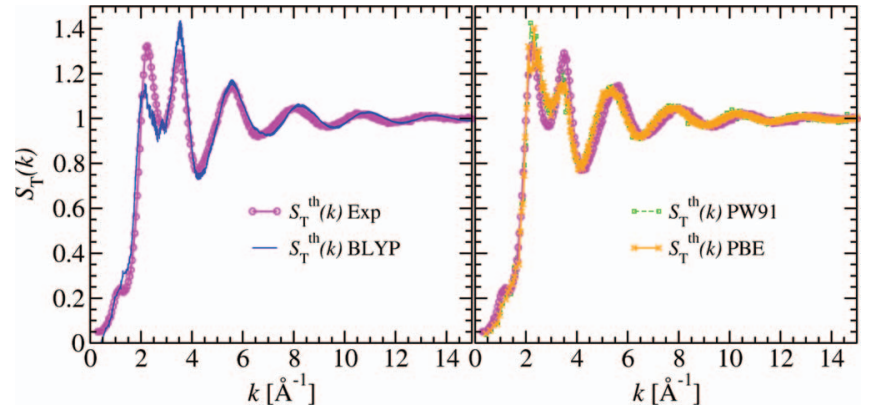

FIG. 1. Total neutron structure factor for liquid GeSe at $T=1000 \mathrm{~K}$. The experimental result $S_{\mathrm{T}}^{\exp }(k)$ given in Ref. 2 (pink line with circles) is compared to $S_{\mathrm{T}}^{\text {th }}(k)_{\mathrm{PW} 91}, S_{\mathrm{T}}^{\text {th }}(k)_{\mathrm{BLYP}}$, and $S_{\mathrm{T}}^{\text {th }}(k)_{\mathrm{PBE}}$.

neutron structure factors do not exactly superpose, none of them reproducing entirely the experimental one for $k \geq 4 \AA^{-1}$.

The experimental, $g_{\mathrm{T}}^{\exp }(r)$, and calculated, $g_{\mathrm{T}}^{\mathrm{PW} 91}(r)$, $g_{\mathrm{T}}^{\mathrm{BLYP}}(r)$, and $g_{\mathrm{T}}^{\mathrm{PBE}}(r)$, total pair distribution functions are shown in Fig. 2. At first sight, $g_{\mathrm{T}}^{\mathrm{PW} 91}(r)$ and $g_{\mathrm{T}}^{\mathrm{PBE}}(r)$ are almost identical and feature remarkable agreement with $g_{\mathrm{T}}^{\exp }(r)$ for both the position and the intensity of the first peak. This same peak is sharper in the $g_{\mathrm{T}}^{\mathrm{BLYP}}(r)$ case, while the first minimum at $r \sim 3 \AA$ is better reproduced. Overall, the BLYP approach leads more structured shells of neighbors, while negligible differences are found, at the total pair distribution level, between the PW91 and the PBE schemes.

\section{RECIPROCAL SPACE PROPERTIES: FABER-ZIMAN PARTIAL STRUCTURE FACTORS}

Among the calculated $S_{\alpha \beta}^{\mathrm{PW} 91}(\mathrm{k}), S_{\alpha \beta}^{\mathrm{BLYP}}(\mathrm{k})$, and $S_{\alpha \beta}^{\mathrm{PBE}}(\mathrm{k})$ Faber-Ziman ${ }^{24}$ partial structure factors shown in Fig. 3, $S_{\mathrm{SeSe}}(\mathrm{k})$ is the one exhibiting the best agreement with the experimental data, $S_{\mathrm{SeSe}}^{\mathrm{exp}}(\mathrm{k})$. Analysis of $S_{\mathrm{SeSe}}^{\mathrm{PW} 91}(\mathrm{k}), S_{\mathrm{SeSe}}^{\mathrm{BLYP}}(\mathrm{k})$, and $S_{\mathrm{SeSe}}^{\mathrm{PBE}}(\mathrm{k})$ reveals that this specific partial structure factor is also the one less sensitive to the XC scheme employed. No substantial differences are found between $S_{\mathrm{SeSe}}^{\mathrm{PBE}}(\mathrm{k})$ and $S_{\mathrm{SeSe}}^{\mathrm{PW}}(\mathrm{k})$. A different situation is encountered with the $S_{\mathrm{GeSe}}(\mathrm{k})$ case. We found that $S_{\mathrm{GeSe}}^{\mathrm{BLYP}}(\mathrm{k})$ differs from $S_{\mathrm{GeSe}}^{\exp }(\mathrm{k}), S_{\mathrm{GeSe}}^{\mathrm{PW}}(\mathrm{k})$ and $S_{\mathrm{GeSe}}^{\mathrm{PBE}}(\mathrm{k})$ in the region $2 \AA^{-1}<k<4 \AA^{-1}$, thereby showing that BLYP describes less well than PBE and PW91 the

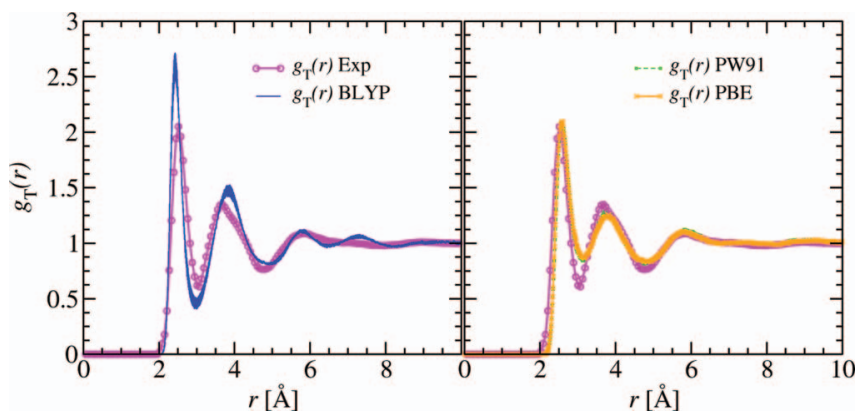

FIG. 2. Total pair distribution function for liquid GeSe at $T=1000 \mathrm{~K}$. The experimental result $g_{\mathrm{T}}^{\exp }(r)$ given in Ref. 2 (pink line with circles) is compared to the calculated functions $g_{\mathrm{T}}^{\text {th }}(r)$ for the PW91, BLYP, and PBE models.

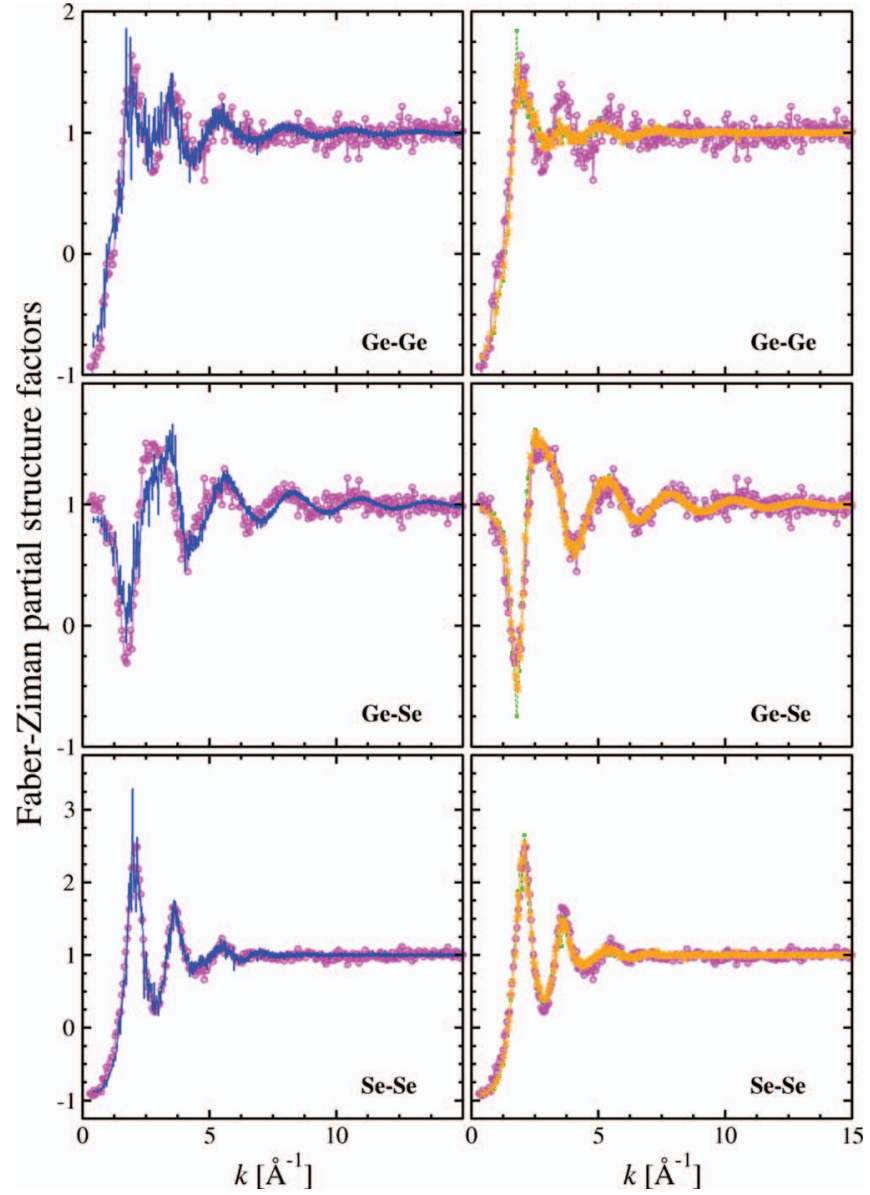

FIG. 3. The Faber-Ziman partial structure factors $S_{\mathrm{GeGe}}(k)$ (top panel), $S_{\mathrm{GeSe}}(k)$ (middle panel), and $S_{\mathrm{SeSe}}(k)$ (bottom panel) for the liquids GeSe. The experimental result given in Ref. 2 (pink line with circles) is compared to the calculated functions for the PW91 (broken green lines with squares), BLYP (blue line), and PBE (orange line with crosses) models.

Ge-Se short range interactions. Also, the prepeak of $S_{\mathrm{GeSe}}^{\mathrm{BLY}}(\mathrm{k})$ is less intense than in the PW91 and PBE counterparts. The $S_{\mathrm{GeGe}}(\mathrm{k})$ partial structure factor is the one that mostly benefits from the BLYP scheme in terms of improved intensities for the maxima and minima, in particular for $k \geq 3.2 \AA^{-1}$. There is a clear contrast between the flattened shape of $S_{\mathrm{GeGe}}^{\mathrm{PW}}(\mathrm{k})$ and $S_{\mathrm{GeGe}}^{\mathrm{PBE}}(\mathrm{k})$ and the distinct pattern of maxima and minima seen in $S_{\mathrm{GeGe}}^{\mathrm{BL}}(\mathrm{k})$. As expected, none of the partial structure factors is characterized by the appearance of a first sharp diffraction peak (FSDP) at about $1 \AA^{-1}$, thereby confirming that the intermediate range order is absent in liquid GeSe.

\section{REAL SPACE PROPERTIES}

\section{A. Pair distribution functions}

Partial pair distribution functions $\mathrm{g}_{\alpha \beta}^{\mathrm{PW} 91}(\mathrm{r}), \mathrm{g}_{\alpha \beta}^{\mathrm{BLYP}}(\mathrm{r})$, and $\mathrm{g}_{\alpha \beta}^{\mathrm{PW} 91}(\mathrm{r})$ are compared to the corresponding experimental data $\mathrm{g}_{\alpha \beta}^{\exp }(\mathrm{r})$ in Fig. 4. The most striking difference between the BLYP scheme and the PW91/PBE ones (once again essentially superposing over the entire $r$ range) lies in the different impact of $\mathrm{Ge}-\mathrm{Ge}$ homopolar bonds. Experimentally, $\mathrm{Ge}-\mathrm{Ge}$ correlations are characterized by a well defined first peak at $2.36 \AA$ (Ref. 25). The BLYP approach improves upon the 


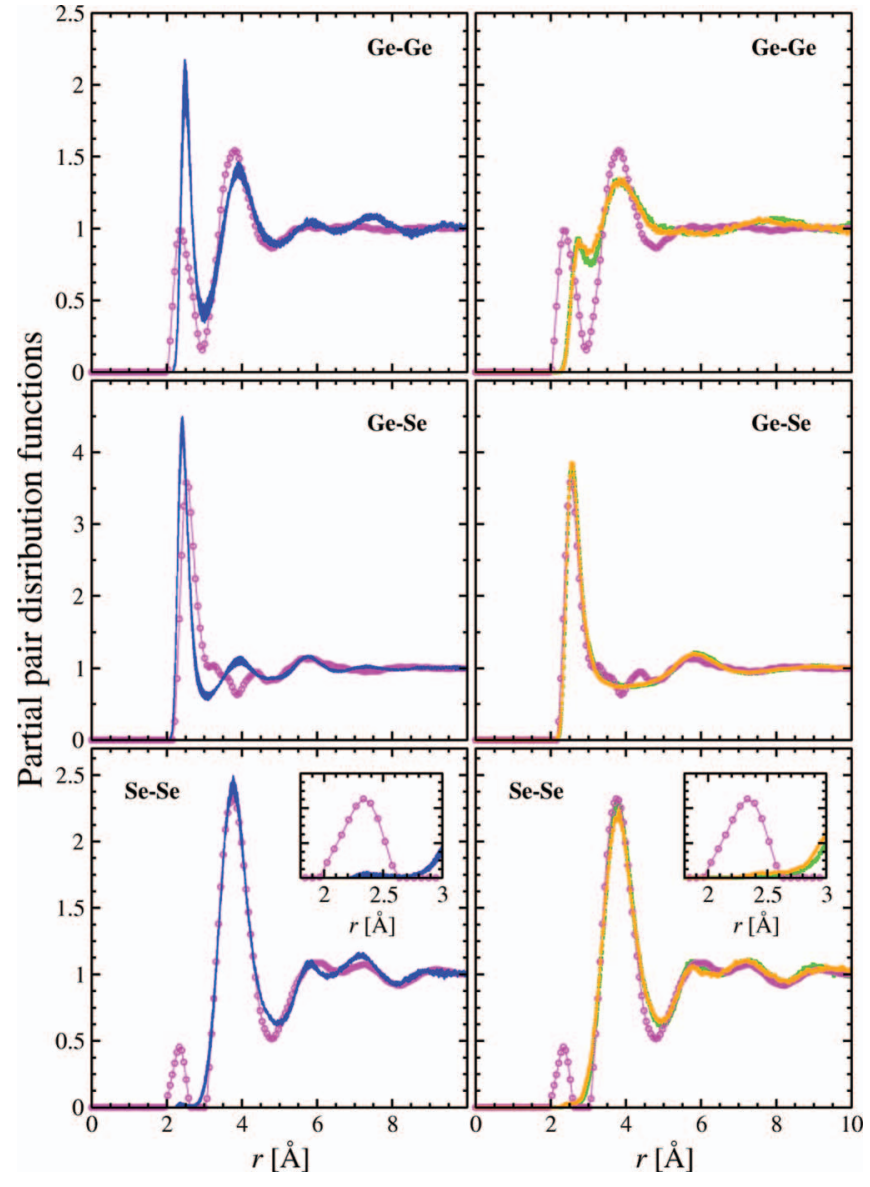

FIG. 4. The partial pair distribution functions $g_{\mathrm{GeGe}}(r)$ (top panel), $g_{\mathrm{GeSe}}(r)$ (middle panel), and $g_{\mathrm{SeSe}}(r)$ (bottom panel) for the liquids GeSe. The experimental result given in Ref. 2 (pink line with circles) is compared to the calculated functions for the PW91 (broken green lines with squares), BLYP (blue line), and PBE (orange line with crosses) models. The insets refer to the $g_{\mathrm{SeSe}}(r)$ partial pair distribution functions in the range comprised between $1.6 \AA$ and $3 \AA$.

PW91 and PBE ones in two respects. First, the maximum in the $\mathrm{g}_{\mathrm{GeGe}}^{\mathrm{BLYP}}(\mathrm{r})$ at short distances is found at $2.49 \AA$, against $2.75-$ $2.77 \AA$ (PBE-PW91). Also, there is clear minimum at $\mathrm{r} \sim 3 \AA$, almost as deep as the experimental one. On the contrary, the intensity of the first peak in $\mathrm{g}_{\mathrm{GeGe}}^{\mathrm{BLY}}(\mathrm{r})$ is much higher than in any of the calculated or measured $\mathrm{Ge}-\mathrm{Ge}$ pair distribution functions, leading to an exceedingly high coordination number for the Ge homopolar interactions (1.2, see Table I). The first peak of $\mathrm{g}_{\mathrm{GeSe}}^{\mathrm{BLY}}(\mathrm{r})$, higher and sharper than in the $\mathrm{g}_{\mathrm{GeSe}}^{\mathrm{PW}}(\mathrm{r})$ and $\mathrm{g}_{\mathrm{GeSe}}^{\mathrm{PBE}}(\mathrm{r})$ cases, exemplifies the higher electronic localization effects typical of this XC functional. A higher level of ionicity in the $\mathrm{Ge}-\mathrm{Se}$ interaction results in a sharper separation between the first two shells of Ge-Se neighbors. Accordingly, $\mathrm{g}_{\mathrm{GeSe}}^{\mathrm{BLYP}}(\mathrm{r})$ features a clear second maximum at $\mathrm{r} \sim 4 \AA$, absent in $\mathrm{g}_{\mathrm{GeSe}}^{\mathrm{PW} 91}(\mathrm{r})$ and $\mathrm{g}_{\mathrm{GeSe}}^{\mathrm{PBE}}(\mathrm{r})$. Minimal differences among the three XC approaches are found at the level of the $\mathrm{g}_{\mathrm{SeSe}}^{\mathrm{PW} 91}(\mathrm{r})$, $\mathrm{g}_{\mathrm{SeSe}}^{\mathrm{PBE}}(\mathrm{r})$ and $\mathrm{g}_{\mathrm{SeSe}}^{\mathrm{BLYP}}(\mathrm{r})$ pair distribution functions, none of them exhibiting a sharp first maximum at $\sim 2.5 \AA$ indicative of SeSe homopolar bonds. However, a few homopolar Se-Se contacts (for distances smaller than $3 \AA$ ) are common to the three approaches, accounting for the tiny features highlighted in the inset of Fig. 4.
TABLE I. The first peak position (FPP) and second peak position (SPP) in $g_{\alpha \beta}(r)$ and the nearest neighbor coordination numbers $\bar{n}_{\alpha \beta}$ obtained from FPMD models of the liquids GeSe (present work). The coordination numbers $\bar{n}_{\alpha \beta}$ for $l$-GeSe were obtained by using an integration range of $0-3.0 \AA$ where the upper limit corresponds to the first minimum in the total pair distribution function. The predictions of the $\mathrm{CON}$ and $\mathrm{RCN}$ models are also listed. ${ }^{2}$

\begin{tabular}{|c|c|c|c|c|c|c|}
\hline$g_{\alpha \beta}(r)$ & Liquid GeSe & $\begin{array}{l}\text { FPP } \\
(\AA)\end{array}$ & $\begin{array}{l}\text { SPP } \\
(\AA)\end{array}$ & $\bar{n}_{\alpha \beta}$ & $\bar{n}_{\alpha \beta}(\mathrm{CON})$ & $\bar{n}_{\alpha \beta}(\mathrm{RCN})$ \\
\hline \multirow[t]{4}{*}{$g_{\mathrm{GeGe}}(r)$} & PW91 & 2.77 & 3.87 & 0.8 & 2 & 2.6667 \\
\hline & BLYP & 2.49 & 3.90 & 1.2 & 2 & 2.6667 \\
\hline & PBE & 2.75 & 3.87 & 0.7 & 2 & 2.6667 \\
\hline & $\exp$ & 2.36 & 3.81 & 0.8 & & \\
\hline \multirow[t]{4}{*}{$g_{\mathrm{GeSe}}(r)$} & PW91 & 2.57 & $\ldots$ & 2.99 & 2 & 1.3333 \\
\hline & BLYP & 2.41 & 3.96 & 2.62 & 2 & 1.3333 \\
\hline & PBE & 2.57 & $\ldots$ & 3.04 & 2 & 1.3333 \\
\hline & $\exp$ & 2.54 & 3.5 & 3.2 & & \\
\hline \multirow[t]{4}{*}{$g_{\mathrm{SeGe}}(r)$} & PW91 & 2.57 & $\ldots$ & 2.99 & 2 & 1.3333 \\
\hline & BLYP & 2.49 & 3.96 & 2.62 & 2 & 1.3333 \\
\hline & PBE & 2.57 & $\ldots$ & 3.04 & 2 & 1.3333 \\
\hline & $\exp$ & 2.54 & 3.5 & 3.2 & & \\
\hline \multirow[t]{4}{*}{$g_{\mathrm{SeSe}}(r)$} & PW91 & 2.50 & 3.80 & 0.1 & 0 & 0.6667 \\
\hline & BLYP & 2.36 & 3.77 & $<0.1$ & 0 & 0.6667 \\
\hline & PBE & 2.46 & 3.82 & $<0.1$ & 0 & 0.6667 \\
\hline & $\exp$ & 2.34 & 3.80 & 0.22 & & \\
\hline
\end{tabular}

\section{B. Coordination numbers}

The coordination numbers $\bar{n}_{\alpha \beta}$ for the liquids GeSe are listed in Table I. They are defined as the mean number of nearest neighbors of type $\beta$ around an atom of type $\alpha$ within an integration range that includes distances up to $3 \AA$, corresponding to the first minimum of the total pair distribution function. In Table I, we report the values of $\bar{n}_{\alpha \beta}$ for two popular models of a network structure, namely, the chemically ordered network $(\mathrm{CON})$ and the random covalent network $(\mathrm{RCN})$. Both of them are compatible with the so-called " $8-\mathrm{N}$ " rule, where $\mathrm{Ge}$ atoms are fourfold coordinated and Se atoms are twofold coordinated. Overall, the BLYP approach does not bring any striking improvement in the coordination numbers and the peak positions, the only relevant exception being the position of the first maximum in $\mathrm{g}_{\mathrm{GeGe}}^{\mathrm{BLYP}}(\mathrm{r})$. Experiments predict total coordination numbers 4 for Ge and 3.44 for Se. Our calculated values are lower for both species, ranging from 3.74 (PBE), 3.79 (PW91) to 3.82 (BLYP) for Ge and from 2.67 (BLYP), 3.07 (PBE) to 3.09 (PW91) for Se. The disagreement is more severe for Se, due to the absence of Se homopolar bonds in our model. Liquid GeSe is characterized by a very peculiar network structure, entirely different from both the CON and the RCN models. This feature was pointed out when comparing the network topology of the liquids $\mathrm{GeSe}_{2}, \mathrm{Ge}_{2} \mathrm{Se}_{3}$, $\mathrm{GeSe}_{4}$, and GeSe, leading to the conclusion that the 8-N rule does not act as an accurate guide to the structure of liquid GeSe. $^{13}$

As an illustration of the network topology in $l$-GeSe we provide in Fig. 5 a snapshot of the structure at a given instant of time along the equilibrium trajectory for the 120 atoms BLYP model. Visual inspection highlights the variety of coordination environments for both $\mathrm{Ge}$ and Se and the lack of 


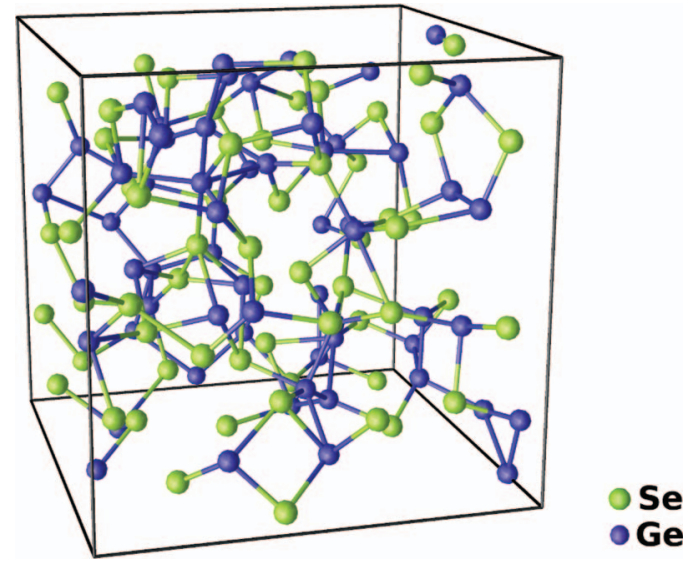

FIG. 5. Snapshot of the structure of the 120 atoms BLYP model for liquid GeSe where the Ge atoms are (dark) blue and the Se atoms are (light) green.

a predominant coordination motif as the chemically ordered $\mathrm{GeSe}_{4}$ tetrahedron.

\section{Structural units and bond angle distributions}

Liquid GeSe can be further described by resorting to the individual $\alpha-l$ structural units where an atom of species $\alpha(\mathrm{Ge}$ or $\mathrm{Se}$ ) is $l$-fold coordinated to other atoms. For instance, $\mathrm{Ge}-$ $\mathrm{GeSe}_{3}$ represents a $\mathrm{Ge}$ atom that is connected to 1 other Ge atom and $3 \mathrm{Se}$ atoms while $\mathrm{Ge}-\mathrm{Se}_{4}$ represents a $\mathrm{Ge}$ atom that is connected to $4 \mathrm{Se}$ atoms. The proportion of a specific unit, $\bar{n}_{\alpha}(l)$, is found by taking the ratio of the mean number of its occurrences to the total number of atoms of type $\alpha$ within the model (Table II). In Ref. 4 it was observed that none of the structural units found in liquid GeSe account for more than $50 \%$ of the coordination environment. In particular, for the case of $l=4$ corresponding to the tetrahedral coordination, this held true not only for a specific $\bar{n}_{\alpha}(l)$ but also when the contributions of the different fourfold coordinations of $\mathrm{Ge}$ (Ge-GeSe $3, \mathrm{Ge}-\mathrm{Ge}_{2} \mathrm{Se}_{2}$, and $\mathrm{Ge}-\mathrm{Se}_{4}$ ) were summed up. This picture is confirmed by the new sets of PW91 and PBE results, in line with the strong similitude between PW91 and PBE results found for the pair distribution functions. At variance with these two functionals, BLYP restores the predominant fourfold character of $\mathrm{Ge}$, since $58.1 \%$ of the Ge atoms have four neighbors. However, high percentages of $\mathrm{Ge}-\mathrm{GeSe}_{3} \mathrm{Ge}-$ $\mathrm{Ge}_{2} \mathrm{Se}_{2}$ units do persist, attaining $24.4 \%$ and $24.2 \%$, respectively. The increase in fourfold coordinated Ge atoms occurs at the expenses of threefold and twofold units (31.3\%, PW91; $33.7 \%$ PBE against $21.4 \%$ BLYP). Concerning the coordinations of the Se atoms, an analogous increase is found for the number of twofold Se atoms $(21.5 \%$, PW91; $18.3 \% \mathrm{PBE}$ against $41.1 \%$ BLYP), even though threefold coordinated Se atoms (close to $50 \%$ of the Se atoms) remain predominant in all the three XC frameworks. One can observe that the larger fraction of tetrahedra goes along with the reduced number of significant structural units, i.e., those accounting for more than $10 \%$ of the coordination environment for a given atomic species. In the present set of BLYP results, this threshold is reached only by threefold and fourfold units (for $\mathrm{Ge}$ ) and by twofold and threefold units (for Se). A larger variety of units
TABLE II. The proportion, $\bar{n}_{\alpha}(l)$ of the different coordination units in the liquids GeSe. The identity of the $\alpha$ atom (Ge or Se) at the center of a unit is given in bold font and the identity of the $l$ nearest neighbors is given in the second column. The cutoff distance has been taken equal to $3 \AA$.

\begin{tabular}{|c|c|c|c|c|}
\hline & & \multicolumn{3}{|c|}{ Proportion $\bar{n}_{\alpha}(l)(\%)$} \\
\hline & & PW91 & BLYP & PBE \\
\hline \multicolumn{5}{|c|}{ Ge atom } \\
\hline \multicolumn{5}{|l|}{$l=2$} \\
\hline & $\mathrm{Se}_{2}$ & 4.8 & 1.8 & 4.0 \\
\hline & $\mathrm{GeSe}$ & 0.6 & 0.5 & 0.4 \\
\hline \multicolumn{5}{|l|}{$l=3$} \\
\hline & $\mathrm{GeSe}_{2}$ & 9.5 & 9.4 & 8.5 \\
\hline & $\mathrm{Se}_{3}$ & 21.8 & 12.0 & 25.2 \\
\hline \multicolumn{5}{|l|}{$l=4$} \\
\hline & $\mathrm{GeSe}_{3}$ & 24.7 & 24.4 & 24.5 \\
\hline & $\mathrm{Ge}_{2} \mathrm{Se}_{2}$ & 7.4 & 24.2 & 6.1 \\
\hline & $\mathrm{Se}_{4}$ & 12.1 & 9.5 & 13.9 \\
\hline \multicolumn{5}{|l|}{$l=5$} \\
\hline & $\mathrm{Ge}_{2} \mathrm{Se}_{3}$ & 5.3 & 4.7 & 4.2 \\
\hline & $\mathrm{GeSe}_{4}$ & 7.0 & 3.2 & 6.7 \\
\hline & $\mathrm{Se}_{5}$ & 2.0 & 0.8 & 2.3 \\
\hline \multicolumn{5}{|c|}{ Se atom } \\
\hline \multicolumn{5}{|l|}{$l=1$} \\
\hline & $\mathrm{Ge}$ & 0.8 & 0.5 & 0.4 \\
\hline & $\mathrm{Se}$ & $<0.1$ & $\ldots$ & $<0.1$ \\
\hline \multicolumn{5}{|l|}{$l=2$} \\
\hline & $\mathrm{Se}_{2}$ & $<0.1$ & $\ldots$ & $<0.1$ \\
\hline & $\mathrm{SeGe}$ & 0.3 & 0.4 & 0.2 \\
\hline & $\mathrm{Ge}_{2}$ & 21.2 & 40.7 & 18.1 \\
\hline \multicolumn{5}{|l|}{$l=3$} \\
\hline & $\mathrm{Se}_{2} \mathrm{Ge}$ & 0.1 & $<0.1$ & 0.1 \\
\hline & $\mathrm{SeGe}_{2}$ & 2.0 & 1.9 & 1.6 \\
\hline & $\mathrm{Ge}_{3}$ & 50.9 & 48.0 & 53.9 \\
\hline \multicolumn{5}{|l|}{$l=4$} \\
\hline & $\mathrm{SeGe}_{3}$ & 2.6 & 1.4 & 2.3 \\
\hline & $\mathrm{Ge}_{4}$ & 19.7 & 6.5 & 20.9 \\
\hline & $\mathrm{Ge}_{2} \mathrm{Se}_{2}$ & 0.2 & $<0.1$ & 0.1 \\
\hline
\end{tabular}

is encountered with the present PW91 and PBE calculations, since fivefold units (for $\mathrm{Ge}$ ) and fourfold units (for $\mathrm{Se}$ ) are also connected to at least $10 \%$ of the Ge or Se atoms. Therefore, electron localization effects inherent in BLYP induce a reduced variability of the coordination number for $\mathrm{Ge}$ and $\mathrm{Se}$ atoms when compared to the PW91 and PBE cases.

The distributions of the Se-Ge-Se and Ge-Se-Ge bond angles are displayed in Fig. 6. We recall that for a predominant tetrahedral network, the $\mathrm{Se}-\mathrm{Ge}-\mathrm{Se}$ bond angle distribution has a maximum around $109^{\circ}$, as it has been observed, for instance, in liquid and glassy $\mathrm{GeSe}_{2} \cdot{ }^{22,23}$ This pattern is not reproduced by PW91 and PBE cases, for which the maximum is found at $90^{\circ}$, in line with the existence of a substantial amount of coordinations differing from the fourfold one. BLYP calculations are expected to restore, at least partially, the typical pattern pertaining to a prototypical tetrahedral network. Indeed, the Se-Ge-Se bond angle distribution moves rightward, with a maximum approaching $100^{\circ}$, clearly distinct from the one corresponding to the PW91 and PBE cases.

Further insight into the connectivity within the network can be gained by examining the $\mathrm{Ge}-\mathrm{Se}-\mathrm{Ge}$ distribution. 


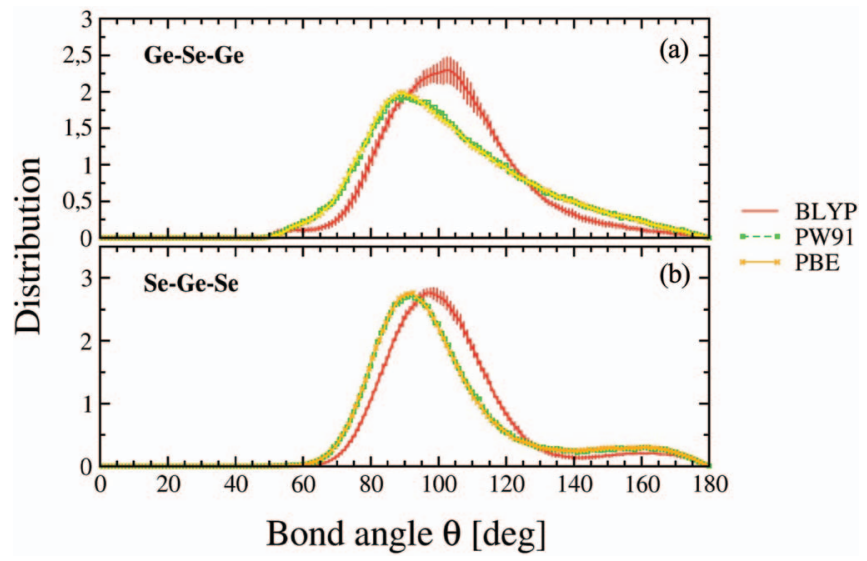

FIG. 6. Angular distribution functions for $\mathrm{Ge}-\mathrm{Se}-\mathrm{Ge}$ (upper panel) and $\mathrm{Se}-$ $\mathrm{Ge}-\mathrm{Se}$ (lower panel) obtained within the three functionals adopted in this work. In both panels the green, yellow, and red curves refer to PW91, PBE, and BLYP, respectively.

Focussing on the PW91 and PBE results, liquid GeSe exhibits a broad maximum, located around $90^{\circ}$, and pronounced tails, these features being indicative of weaker directional bonds. BLYP introduces a displacement of the distribution toward higher angles, with the maximum attained for $\sim 100^{\circ}$ and a perceptible large bump on the left part of the bell-shaped pattern. These are fingerprints of uncomplete and yet, sizeable organization around the Ge centers, involving edge-sharing (at around $80^{\circ}-90^{\circ}$ ) and corner sharing (close to $100^{\circ}$ ) connections, as shown in the past for the case of the strongly tetrahedral networks liquid and glassy $\mathrm{GeSe}_{2} \cdot{ }^{10,22}$ The above considerations further exemplify the role played by the BLYP approach in the establishment of a more pronounced tetrahedral character within liquid GeSe.

\section{DIFFUSION AND DYNAMICAL PROPERTIES}

Diffusion is a key physical property to understand how the atomic structure affects the mobility of a given network. In the past, the marked increase in the tetrahedral order of liquid $\mathrm{GeSe}_{2}$, obtained with the BLYP approach, was found to be the cause of a substantial decrease in the diffusion coefficient when compared to the PW91 case (see Ref. 10). These different behaviors could be ascribed to the enhanced mobility of miscoordinated Ge-centered units (common in the PW91 case), this same mobility being significantly reduced when the number of chemically ordered tetrahedra increases (BLYP case). In order to assess the impact of the XC choice on the diffusion properties of liquid GeSe, we have calculated the statistical average of the mean square displacement of chemical species $\alpha$

$$
\left\langle r_{\alpha}^{2}(t)\right\rangle=\frac{1}{N_{\alpha}}\left\langle\sum_{i=1}^{N_{\alpha}}\left|\mathbf{r}_{i \alpha}(t)-\mathbf{r}_{i \alpha}(0)\right|^{2}\right\rangle,
$$

where $\mathbf{r}_{i \alpha}(t)$ is the coordinate of the $i$ th particle of chemical species $\alpha$ at time $t$ and $N_{\alpha}$ is the total number of particles of type $\alpha$. The mean square displacements calculated for the $\mathrm{Ge}$ and Se atoms are shown in the inset to Fig. 7. Provided the diffusive regime is attained, the diffusion coefficient is given

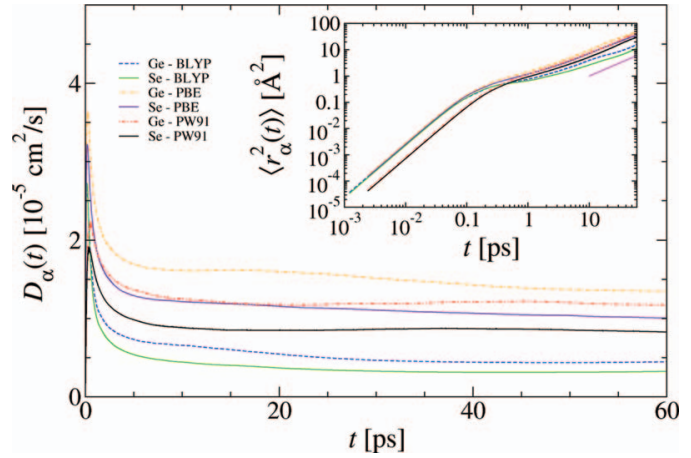

FIG. 7. The diffusion coefficients of Ge atoms (broken lines) and Se atoms (solid lines) in liquid GeSe at $T=1000 \mathrm{~K}$ as calculated from the mean square displacement of a given chemical species at time $t$ (see Eq. (4)). The inset shows the time dependence of the mean square displacements of the Ge atoms (broken lines) and the Se atoms (solid lines), and the straight pink line indicates the slope corresponding to the diffusive regime.

by the typical Einstein's relation

$$
D_{\alpha}=\frac{\left\langle r_{\alpha}^{2}(t)\right\rangle}{6 t}
$$

The plot of $\log \left\langle r_{\alpha}^{2}(t)\right\rangle$ vs $\log t$ shows linear behavior in the long time limit with a gradient equal to unity. The values of $D_{\mathrm{Ge}}$ and $D_{\mathrm{Se}}$ obtained in this work (PW91, BLYP, and PBE approaches) are collected in Table III and compared to those of Ref. 10 (liquid $\mathrm{GeSe}_{2}$ ) and liquid $\mathrm{Ge}_{2} \mathrm{Se}_{3}{ }^{13}$ (BLYP calculations in the two latter cases). Care must be exercised when comparing the PW91 values resulting from the new trajectory with those reported in Ref. 4 for the previous set of PW91 calculations. The difference lies in the time interval considered to extrapolate the infinite time behavior (i.e., diffusive regime) of the mean square displacement. In this work, we have been able to capture the extended relaxation times that characterize diffusion, by exploiting a fairly long temporal trajectory (100 ps for the entire run, with averages taken for the first 60 ps, see Fig. 7). This results in a marked decrease of the diffusion coefficient when compared to the value of Ref. 4, extracted from a temporal behavior limited to $5 \mathrm{ps}$.

In view of the statistical accuracy, the BLYP values of $D_{\mathrm{Ge}}$ and $D_{\mathrm{Se}}$ for liquid $\mathrm{Ge}_{2} \mathrm{Se}_{3}$ and liquid GeSe are not significantly different. However, both of them are larger than in liquid $\mathrm{GeSe}_{2}$. Liquid $\mathrm{Ge}_{2} \mathrm{Se}_{3}$ and liquid GeSe lie on the Gerich side of the $\mathrm{Ge}_{x} \mathrm{Se}_{1-x}$ composition range and share the presence of $\mathrm{Ge}$ atoms not tetrahedrally coordinated, prone to a larger mobility than in the stoichiometric $\mathrm{GeSe}_{2}$ case. In

TABLE III. The diffusion coefficients of the Ge and Se atoms in the liquids GeSe as obtained from FPMD models.

\begin{tabular}{lcc}
\hline \hline & \multicolumn{2}{c}{$D_{\alpha}\left(\times 10^{-5} \mathrm{~cm}^{2} / \mathrm{s}\right)$} \\
\cline { 2 - 3 } & $\mathbf{G e}$ & $\mathbf{S e}$ \\
\hline$l-\mathrm{GeSe}_{2}$ (Ref. 10) & $0.2 \pm 0.2$ & $0.2 \pm 0.2$ \\
$l$-Ge $\mathrm{Se}_{3}$ (Ref. 13) & $0.62 \pm 0.2$ & $0.46 \pm 0.2$ \\
$l$-GeSe PW91 - 108 atoms (present work) & $1.20 \pm 0.1$ & $0.85 \pm 0.1$ \\
$l$-GeSe BLYP - 120 atoms (present work) & $0.50 \pm 0.1$ & $0.35 \pm 0.1$ \\
$l$-GeSe PBE - 120 atoms (present work) & $1.48 \pm 0.2$ & $1.0 \pm 0.1$ \\
\hline
\end{tabular}




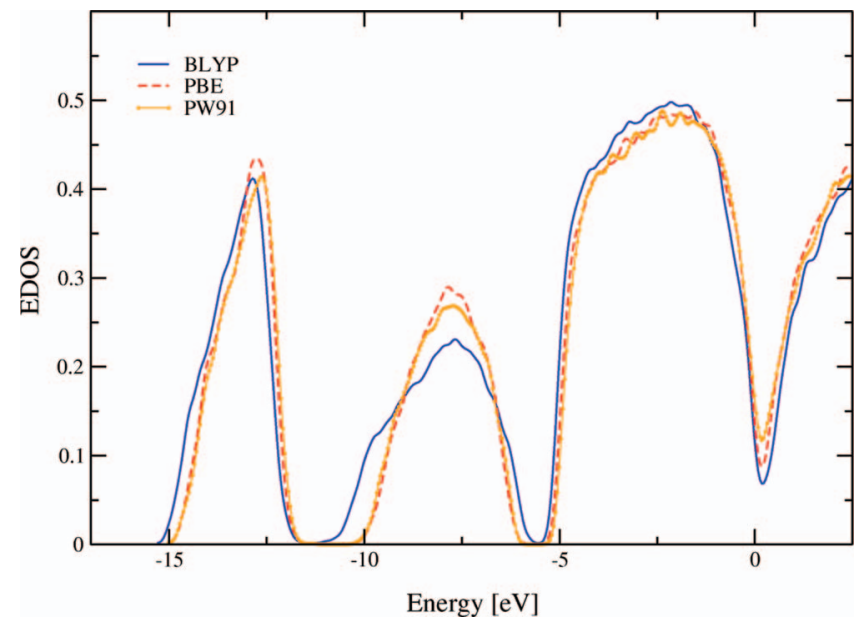

FIG. 8. Electronic density of states for the BLYP (blue line), PBE (dashed red line), and PW91 (orange line with symbols) models of liquid GeSe at $T$ $=1000 \mathrm{~K}$. A Gaussian broadening of $0.1 \mathrm{eV}$ has been employed.

addition, $\mathrm{Ge}-\mathrm{Ge}$ homopolar bonds alter the regularity of a the $\mathrm{GeSe}_{4}$ tetrahedron-based network, favoring atomic diffusion. We note that the diffusion coefficient is drastically reduced for both species in the BLYP framework, in line with what found for liquid $\mathrm{GeSe}_{2}$. Once again, this can be ascribed to the increase of the tetrahedral coordination and the reduced variety of structural units accounting for the Ge and Se coordination environments. The better agreement with experiments found in the BLYP case for liquid $\mathrm{GeSe}_{2}$ suggest that the present BLYP results provide a reliable estimate of the diffusion coefficients of liquid GeSe. This assertion has, at least, a plausible character in the absence of experimental diffusion data.

\section{ELECTRONIC DENSITY OF STATES}

In recent papers, we have established clear correlations between structural and electronic properties of disordered chalcogenides. ${ }^{10,13,26}$ For instance, in the case of $l$-GeSe 2 , we have compared the performances of two DFT models that differed in the exchange-correlation part of the DFT functional. ${ }^{10} \mathrm{~A}$ clear correlation was found between increased tetrahedral ordering and the deeper pseudogap around the Fermi level in the electronic density of states (EDOS) when considering PW91 and BLYP models. Also, we have addressed the issue of the existence of such a correlation when the tetrahedral order is modified by a change in concentration within the family of $\mathrm{Ge}_{x} \mathrm{Se}_{1-x}$ liquids and glasses. ${ }^{13,26}$ For instance, in the case of the $\mathrm{Ge}_{2} \mathrm{Se}_{3}$ networks, the EDOS of glassy $\mathrm{Ge}_{2} \mathrm{Se}_{3}$ has a much deeper pseudogap around the Fermi level than the corresponding liquid, indicating that frequent bond breaking inherent in the liquid is associated with the enhanced metallic character.

Here we focus on a comparative analysis of the EDOS for the three models of liquid GeSe detailed above (see Figure 8). For the three cases, 50 independent configurations were extracted from the whole equilibrium trajectory and were used in making the statistical averages. The larger percentage of tetrahedra and the decrease in the diffusion coefficient are indicative of a more important role of the iono-covalent char- acter of bonding over the metallic one. Such trend correlates well with the deepest pseudogap observed in the BLYP case. This is particularly striking when the PW91 and the BLYP are examined. On the contrary, the deeper pseudogap of the PBE EDOS does not have an unambiguous counterpart in any of the corresponding structural properties, especially when compared to the those found by using the PW91 scheme.

\section{CONCLUSION}

The description of bonding in disordered chalcogenides is crucially dependent on the performances of the exchangecorrelation functional implemented within the DFT KohnSham formalism. In the past, the significance of this assertion has been demonstrated for the case of $\mathrm{GeSe}_{2}$ systems by calculating an extensive set of structural and dynamical properties within the PW91 and the BLYP schemes. ${ }^{10}$ The stronger electronic localization associated to this latter functional led to an improved description of short range properties and atomic mobility. Along the same lines, the PBE functional has been selected as an alternative to the PW91 scheme to investigate glassy $\mathrm{GeSe}_{4} \cdot{ }^{11} \mathrm{In}$ that case, only moderate changes were found when comparing the PW91 and the PBE results.

In view of these findings, it appears useful to assess the performances of the BLYP and of the PBE schemes when applied to the case of liquid GeSe. Previous PW91 results on liquid GeSe were characterized by non-negligible differences between measured and calculated partial pair distribution functions $\mathrm{g}_{\mathrm{GeGe}}(\mathrm{r})$ and $\mathrm{g}_{\mathrm{SeSe}}(\mathrm{r}){ }^{4}$ Focussing on the PBE results, no relevant differences are recorded between the new set of PBE data and the previous PW91 ones. This indicates that for this specific bonding topology, the ingredients introduced in the PBE scheme to go beyond the PW91 recipe have a very limited impact on the structural properties. In the past, the close performances of the PW91 and the PBE approaches were underlined for the case of liquid gallium and of water dissociation on the $\mathrm{Cu}(111)$ substrate. ${ }^{27,28}$

The present results do confirm the propensity of the BLYP approach to better describe Ge-Ge short range correlations, since two clear maxima separated by a sharp minimum are found in the $\mathrm{g}_{\mathrm{GeGe}}^{\mathrm{BLP}}(\mathrm{r})$ pair distribution function. Also, the number of tetrahedral connections is increased, by conferring to the network an enhanced stability which, in turn, results in a significant decrease of the diffusion coefficients for both species. This feature appears as the most significant improvement due to the BLYP scheme. In spite of these progresses, we have to mention some drawbacks arising from the electron localization tendency inherent in the BLYP scheme. In particular, the Ge-Se short range shell of interactions is overstructured, leading to a two peaks pattern in $\mathrm{g}_{\mathrm{GeSe}}^{\mathrm{BLYP}}(\mathrm{r})$ for short distances. Also, the strong intensity of the first peak in $\mathrm{g}_{\mathrm{GeGe}}^{\mathrm{BLYP}}(\mathrm{r})$ results in coordination numbers only in fair agreement with experiments. Overall, the quest for an optimal description of bonding in $\mathrm{Ge}-\mathrm{Se}$ chalcogenides remains as an open challenge, calling for the use of more complex functional schemes expected to better describe the transition between iono-covalent and metallic bonding for increasing $\mathrm{Ge}$ content. 
${ }^{1}$ P. S. Salmon, R. A. Martin, P. E. Mason, and G. J. Cuello, Nature (London) 435, 75 (2005).

${ }^{2}$ P. S. Salmon, J. Non-Cryst. Solids 353, 2959 (2007).

${ }^{3}$ C. Massobrio, M. Celino, P. S. Salmon, R. A. Martin, M. Micoulaut, and A. Pasquarello, Phys. Rev. B 79, 174201 (2009).

${ }^{4}$ F. H. M. van Roon, C. Massobrio, E. de Wolff, and S. W. de Leeuw, J. Chem. Phys. 113, 5425 (2000).

${ }^{5}$ J. Y. Raty, V. V. Godlevsky, J. P. Gaspard, C. Bichara, M. Bionducci, R. Bellissent, R. Ceolin, J. R. Chelikowsky, and P. Ghosez, Phys. Rev. B 64, 235209 (2001)

${ }^{6}$ J. P. Perdew and Y. Wang, Phys. Rev. B 45, 13244 (1992).

${ }^{7}$ J. P. Perdew, J. A. Chevary, S. H. Vosko, K. A. Jackson, M. R. Pederson, D. J. Singh, and C. Fiolhais, Phys. Rev. B 46, 6671 (1992).

${ }^{8}$ A. D. Becke, Phys. Rev. A 38, 3098 (1988).

${ }^{9}$ C. Lee, W. Yang, and R. G. Parr, Phys. Rev. B 37, 785 (1988).

${ }^{10}$ M. Micoulaut, R. Vuilleumier, and C. Massobrio, Phys. Rev. B 79, 214205 (2009).

${ }^{11}$ K. Sykina, E. Furet, B. Bureau, S. Le Roux, and C. Massobrio, Chem. Phys. Lett. 547, 30 (2012).

${ }^{12}$ M. Bauchy, M. Micoulaut, M. Celino, S. Le Roux, M. Boero, and C. Massobrio, Phys. Rev. B 84, 054201 (2011).

${ }^{13}$ S. Le Roux, A. Zeidler, P. S. Salmon, M. Boero, M. Micoulaut, and C. Massobrio, Phys. Rev. B 84, 134203 (2011).
${ }^{14}$ M. Boero and W. Andreoni, Chem. Phys. Lett. 265, 24 (1997).

${ }^{15}$ J. P. Perdew, K. Burke, and M. Ernzerhof, Phys. Rev. Lett. 77, 3865 (1996).

${ }^{16}$ J. Akola and R. O. Jones, Phys. Rev. Lett. 100, 205502 (2008).

${ }^{17}$ G. C. Sosso, S. Caravati, R. Mazzarello, and M. Bernasconi, Phys. Rev. B 83, 134201 (2011).

${ }^{18}$ G. C. Sosso, G. Miceli, S. Caravati, J. Behler, and M. Bernasconi, Phys. Rev. B 85, 174103 (2012).

${ }^{19}$ R. Car and M. Parrinello, Phys. Rev. Lett. 55, 2471 (1985).

${ }^{20}$ J. Sarnthein, A. Pasquarello, and R. Car, Phys. Rev. B 52, 12690 (1995).

${ }^{21}$ N. Troullier and J. L. Martins, Phys. Rev. B 43, 1993 (1991).

${ }^{22}$ C. Massobrio and A. Pasquarello, Phys. Rev. B 77, 144207 (2008).

${ }^{23}$ C. Massobrio, A. Pasquarello, and R. Car, Phys. Rev. B 64, 144205 (2001).

${ }^{24}$ Y. Waseda, The Structure of Non-Crystalline Materials (McGraw-Hill, New York, 1980), describes the relationship between the three sets of partial structure factors commonly used (Faber-Ziman, Ashcroft-Langreth, and Bhatia-Thornton).

${ }^{25}$ I. Petri, P. S. Salmon, and H. E. Fischer, J. Phys.: Condens. Matter 11, 7051 (1999).

${ }^{26}$ S. Le Roux, A. Bouzid, M. Boero, and C. Massobrio, Phys. Rev. B 86, 224201 (2012)

${ }^{27}$ J. L. C. Fajín, F. Illas, and J. R. B. Gomes, J. Chem. Phys. 130, 224702 (2009).

${ }^{28}$ J. Yang, J. S. Tse, and T. Iitaka, J. Chem. Phys. 135, 044507 (2011). 